\title{
The Prognostic Significance of miR-21 Expression among Surgically Resected Hepatocellular Carcinoma Patients: Evidence from a Meta-Analysis and Retrospective Cohort Study
}

\author{
Xujian Huang $\mathbb{D}^{1,2}$ Yongfu Xiong, ${ }^{1,2}$ Jialin Yang, ${ }^{3}$ Gang Yang, ${ }^{1}$ and Jingdong $L i \mathbb{D}^{1,2}$ \\ ${ }^{1}$ Department of Hepatobiliary Surgery, Affiliated Hospital of North Sichuan Medical College, Nanchong 637000, China \\ ${ }^{2}$ Institute of Hepato-Biliary-Pancreatic-Intestinal Disease, North Sichuan Medical College, Nanchong 637000, China \\ ${ }^{3}$ Medical Imaging Center, Nanchong Central Hospital/Second School of Clinical Medicine, North Sichuan Medical College, \\ Nanchong 637000, China
}

Correspondence should be addressed to Jingdong Li; li-jingdong@hotmail.com

Received 31 August 2020; Accepted 23 November 2020; Published 21 December 2020

Academic Editor: Kyoung-Ho Pyo

Copyright (C) 2020 Xujian Huang et al. This is an open access article distributed under the Creative Commons Attribution License, which permits unrestricted use, distribution, and reproduction in any medium, provided the original work is properly cited.

Background. To date, microRNA-21 (miR-21) has been reported to be associated with the prognosis of hepatocellular carcinoma (HCC) in various studies, yet the results were inconsistent. The purpose of this two-part study, consisting of a retrospective cohort study and a meta-analysis, sets out to determine the prognostic role of miR-21 expression among HCC patients who underwent surgical resection. Methods. In this study, we first detected miR-21 expression in HCC patients by quantitative real-time PCR (qRT-PCR). Patients were divided into a high miR-21 expression group and a low miR-21 expression group according to the median level of miR-21 expression in tumor tissues. The survival outcomes of the two groups were analyzed by the Kaplan-Meier method with the log-rank test. Multivariate analysis of the prognostic factors was performed with the Cox regression model. Subsequently, eligible studies were obtained by searching on PubMed, Cochrane Library, and Web of Science, and a meta-analysis was performed to assess the prognostic role of miR-21 expression among HCC. Results. The qRT-PCR analysis results of our cohort study showed miR-21 expression was significantly upregulated in HCC tissues when compared with adjacent nontumor tissues. Multivariate analysis suggested that miR-21 expression was an independent prognostic factor for overall survival (OS) (hazard ratio, $\mathrm{HR}=2.361$ ) and disease-free survival (DFS) $(\mathrm{HR}=2.024)$ in $\mathrm{HCC}$ patients who underwent surgical resection. A total of 10 studies with 969 patients were enrolled in the meta-analysis, consisting of 9 studies from the database search and our cohort study. We observed that elevated miR-21 expression can predict poor OS $(\mathrm{HR}=2.24$, $95 \% \mathrm{CI}=1.73-2.91, P<0.001)$ and $\mathrm{DFS} /$ recurrence-free survival $(\mathrm{RFS})(\mathrm{HR}=2.44,95 \% \mathrm{CI}=1.62-3.67, P<0.001)$ in surgically resected HCC patients. Conclusions. Our study demonstrated that miR-21 high expression among surgically resected HCC patients is a prognostic factor that indicated adverse survival.

\section{Introduction}

Liver cancer is one of the most common cancers in the world, results in 841,000 new cases per year, and kills 782,000 people annually $[1,2]$. Hepatocellular carcinoma (HCC) is the most frequent type, accounting for $75 \%-85 \%$ of all primary liver cancers, and is the third leading cause of malignant tumorrelated deaths, with increasing incidence each year [3]. Radical resection is the main method for the treatment of liver cancer [4]. In recent years, with the accumulation of surgical experience, the application of advanced instruments, and the progress of perioperative evaluation and management, the 5 -year survival rate after hepatectomy has increased, but the oncology prognosis of patients with HCC is still unfavorable because of its biological characteristics such as rapid invasive growth, poor differentiation, and early metastasis [5-7]. Therefore, it is crucial to identify prognostic markers which can identify different phenotypes with differences in 


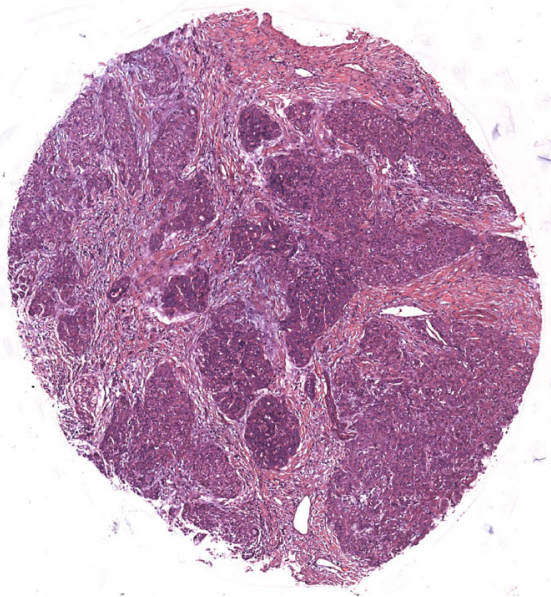

(a)

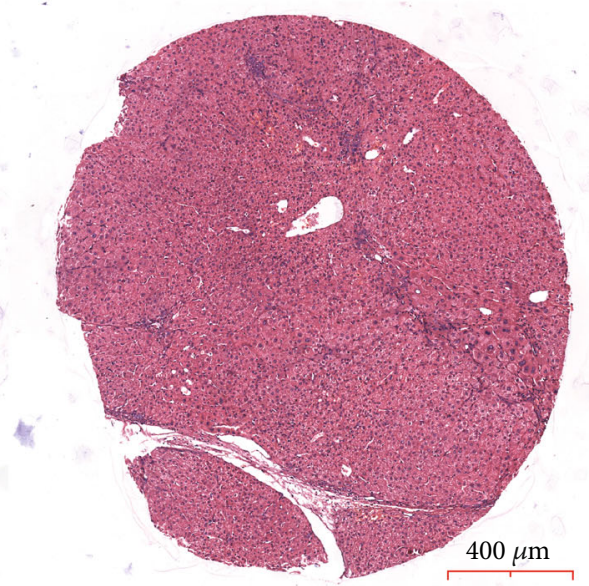

(b)

FIgURE 1: H\&E staining of HCC specimen (a) and adjacent nontumor tissue (b).

clinical characteristics and prognosis of HCC for guiding clinical management.

MicroRNAs (miRNAs) are a large family of small, approximately 21-nucleotide-long, noncoding RNAs that are predicted to control the activity of approximately $30 \%$ of all protein-coding genes and have been shown to participate in the regulation of almost every cellular process in mammals $[8,9]$. More importantly, recent studies suggest that microRNAs also play a significant role in carcinogenesis acting either as oncogenes or tumor suppressors $[10,11]$. miR-21 is one of the most frequently observed cancerrelated microRNAs [9, 12, 13]. Previous studies have observed that miR-21 elevates in many cancers such as breast cancer, lung cancer, pancreatic cancer, stomach cancer, and hepatocellular carcinomas and acts as a key factor mediating the growth, development, and progression of tumors $[10,14-16]$. Recent reports have suggested that miR-21 expression is related to survival in HCC patients $[12,13,17,18]$; however, the evidence remains inconclusive. Thus, we conducted a two-part study comprising a retrospective cohort study and a meta-analysis to further explore the prognostic significance of miR-21 expression in HCC patients who underwent surgical resection.

\section{Materials and Methods}

2.1. Study Population. This study was approved by the Research Ethics Committee of the Affiliated Hospital of North Sichuan Medical College, and informed consent was provided by all 166 patients with HCC who underwent radical hepatectomy from January 2013 to December 2015. According to the median level of miR-21 expression in cancer tissues, these patients were divided into a high miR-21 expression group and a low miR-21 expression group. The diagnosis of HCC was confirmed by clinical resection and pathology. Patients who received neoadjuvant chemotherapy, patients with distant metastases during surgery, and patients with other malignancies were excluded from this study. The operation was performed by several surgeons according to the standard criteria for hepatectomy in our hospital.

2.2. Histopathological Evaluation. Histopathological evaluation of all surgical specimens was fulfilled based on standard path procedures, and hematoxylin and eosin- (H\&E-) stained slides from all cases were reviewed by hepatic pathologists without any knowledge of the clinical outcomes (Figure 1).

2.3. RNA Isolation and $q R T-P C R$. Total RNA was extracted from the surgically resected tissues by using the TRIzol RNA extraction kit. cDNA was generated using the PrimeScript RT reagent kit (Takara) in a $20 \mu$ l final reaction volume containing $0.5 \mu \mathrm{g}$ of RNA, $0.5 \mu \mathrm{l}$ PrimeScript RT enzyme mix, $4 \mu \mathrm{l} 5 \mathrm{x}$ PrimeScript buffer, and $1 \mu \mathrm{l}$ RT primer and incubated at $42^{\circ} \mathrm{C}$ for $60 \mathrm{~min}$ and at $85^{\circ} \mathrm{C}$ for $5 \mathrm{~min}$. The quantitative real-time PCR assay was performed to evaluate miR-21 expression using SYBR Premix Ex Taq (Takara) and measured in a LightCycler 480 System (Roche). The amplification profile was denatured at $95^{\circ} \mathrm{C}$ for $10 \mathrm{~min}$, followed by 45 cycles of denaturation at $95^{\circ} \mathrm{C}$ for $15 \mathrm{~s}$, annealing at $60^{\circ} \mathrm{C}$ for $30 \mathrm{~s}$, and extension at $72^{\circ} \mathrm{C}$ for $1 \mathrm{~min}$. The relative expression of miR-21 was calculated and normalized using the $2^{-\Delta \Delta \mathrm{Ct}}$ method relative to U6 small nuclear RNA.

\section{Statistical Analysis}

Using SPSS25.0 statistical software (SPSS Inc., Chicago, USA) to evaluate the prognostic significance of miR-21. Relationships between miR-21 expression and patient characteristics were investigated using Pearson's $\chi^{2}$ test and Spearman's correlation analysis. The differences in overall survival (OS) and disease-free survival (DFS) between the two groups were analyzed using the Kaplan-Meier method with the log-rank test. Univariate analysis was used to establish the potential prognostic factors for OS and DFS, and multivariate analysis for significant factors was performed by Cox proportional hazard regression models. A $P$ value of $<0.05$ indicated a statistically significant difference. 


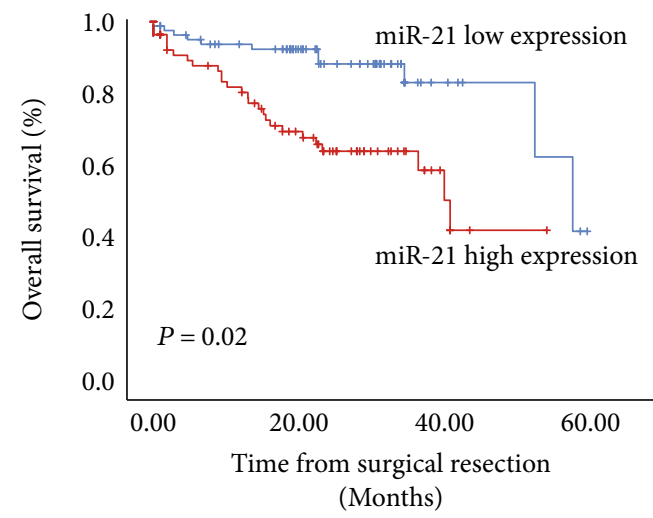

(a)

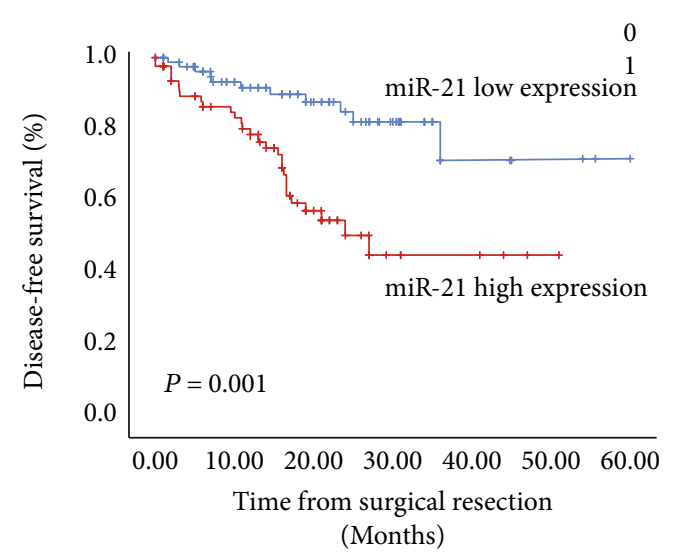

(b)

FIgURE 2: Kaplan-Meier curves for overall survival (a) and disease-free survival (b) according to miR-21 expression in HCC patients who underwent surgical resection.

\section{Meta-Analysis}

This meta-analysis was performed in accordance with the guidelines of the Preferred Reporting Items for Systematic Reviews and Meta-Analyses (PRISMA) [19].

4.1. Search Strategy. We performed a systematic literature search on PubMed, Cochrane Library, and Web of Science databases for articles that assessed the relationship between miRNA-21 and the prognosis of HCC patients who underwent radical resection until December 31, 2019, and with no lower date limit. The following terms were used: (1) "microRNA-21" or "miR-21" or miRNA-21, and (2) "carcinoma*, hepatocellular" or "hepatocellular carcinoma*" or "hepatoma*" or "liver cell carcinoma*" or carcinoma*, liver cell, and (3) "prognos"” or "survival" or "outcome".

4.2. Selection Criteria. All of the eligible studies met the following criteria: (i) studied patients with HCC based on histopathological confirmation, (ii) studied patients with HCC underwent surgical resection, and (iii) investigated the survival outcome or the correlation between miR-21 expression and the clinical characteristics. Studies were excluded based on any of the following criteria: (i) reviews, letters, and case reports; (ii) non-English studies; (iii) studies had overlapping or duplicate data; and (iv) lacked key information for calculation with methods established by Parmar et al., Williamson et al., and Tierney et al. [20-22]. A flow diagram of the study selection process is summarized in Figure 2.

4.3. Quality Assessment. The Newcastle-Ottawa Scale [23] was used to evaluate the quality of each study included in the meta-analysis and three aspects were generally assessed: population selection, study comparability, and reporting of the outcome, with a score ranging from 0 to 9 . A study with a score of $\geq 6$ was considered to be of high quality.

4.4. Data Extraction. Two investigators (Huang Xujian and Yang Jialin) independently reviewed each eligible study and extracted data from studies following the guidelines before mentioned selection criteria [24]. The controversial issues were resolved by discussion, and consensus was reached by the third investigator (Li Jingdong) when there were disagreements between two investigators. The following details were summarized from each eligible study: first author's name, published year, country, ethnicity, sample size, patients' sex, detection method, cutoff value, HRs with 95\% confidence intervals (CIs), and $P$ value of miR-21 for OS and DFS/RFS. If only survival curves were available, data were extracted using the method described by Tierney et al. [21].

4.5. Statistical Methods. Pooled hazard ratio (HR) with $95 \%$ CI was used to evaluate the relation between high miR-21 expression and prognosis of HCC patients. Pooled HR $>1$ implied unfavorable prognosis for the groups with elevated miR-21 expression and had statistical significance if the 95\% CI did not overlap 1, while $\mathrm{HR}<1$ implied a favorable prognosis. The heterogeneity was assessed using $I^{2}$ statistic $\left(P<0.1\right.$ or $I^{2}>50 \%$ indicate significant heterogeneity) described by Higgins et al. [25]. The random-effects model was used if significant heterogeneity exists among studies; otherwise, the fixed-effects model was used. Publication bias was assessed using the funnel plot. Forrest plots were used to estimate the effect of miR-21 expression on survival outcomes (OS and DFS/RFS). Data analyses were performed using RevMan software, version 5.3 (the Nordic Cochrane Centre, Cochrane Collaboration, Copenhagen, Denmark) and Stata 14.0 (Stata Corporation, College Station, TX, USA). $P<0.05$ denoted statistical significance.

\section{Results}

5.1. The Expression of miR-21 Is Significantly Upregulated in Human HCC Tissues. The qRT-PCR assay was performed to determine the expression level of miR-21 in 166 cases of HCC and their matched nontumor tissues. The results showed that the expression of miR-21 in hepatocellular carcinoma was significantly higher than that in nontumor tissues $(P<0.05$, Figure 3). Based on the median level of miR-21 in cancer tissues, 166 HCC patients were divided into a high 


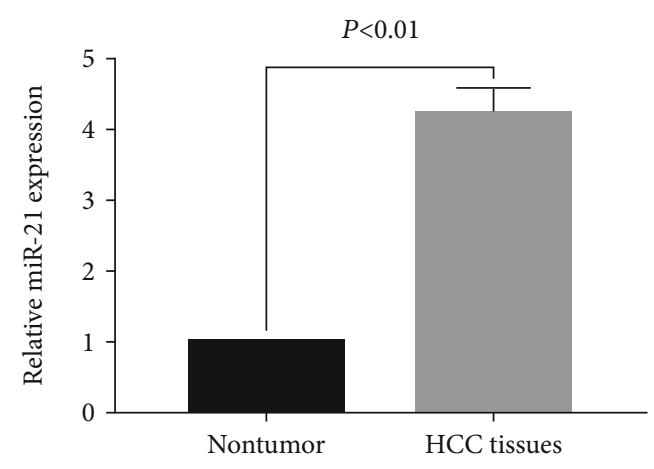

FIGURE 3: Relative expression levels of miR-21 in 166 paired HCC and adjacent nontumor tissues.

miR-21 expression group $(n=83)$ and a low miR-21 expression group $(n=83)$ (Table 1$)$.

\subsection{Association of miR-21 Expression with Clinic-Pathological} Characteristics of HCC Patients. For a better understanding of the clinical significance of miR-21 expression in HCC, we further investigated the clinic-pathological characteristics of HCC. As shown in Table 1, the high expression level of miR-21 was closely related to tumor size, tumor number, tumor differentiation, and TNM stage $(P<0.05)$. Nevertheless, there were no associations between the expression of miR-21 and the other clinic-pathological parameters including age, sex, vascular infiltration, $\alpha$-fetoprotein (AFP) level, and cirrhosis $(P>0.05)$ (Table 1$)$.

5.3. The Impact of miR-21 Expression on OS in HCC. To determine whether the miR-21 expression level is a significant predictor for OS after hepatectomy, the Kaplan-Meier OS curve of the HCC patients according to the status of the miR-21 level was examined. The OS of patients with high miR-21 expression was significantly lower than those with low miR-21 expression $(P=0.02$; Figure 2(a)). Univariate analysis showed that tumor size, tumor number, tumor differentiation, vascular infiltration, cirrhosis, TNM stage, and high miR-21 expression were related to a poor overall survival for patients with HCC. Further multivariate analysis showed that high miR-21 expression $(\mathrm{HR}=2.361 ; 95 \% \mathrm{CI}=$ 1.016-5.490; $P=0.046)$, vascular infiltration $(\mathrm{HR}=2.56$; $95 \% \mathrm{CI}=1.162-5.639 ; P=0.019)$, and tumor size $(\mathrm{HR}=$ 3.902; $95 \% \mathrm{CI}=1.273-11.956 ; P=0.017)$ were independent prognostic factors for OS. The results are shown in Table 2.

5.4. The Impact of miR-21 Expression on DFS in HCC. Kaplan-Meier analysis demonstrated that DFS was significantly shorter in the miR-21 high expression group compared with the miR-21 low expression group $(P=0.001$, Figure 2(b)). In addition, univariate Cox regression analysis indicated that miR-21 expression, tumor number, tumor differentiation, vascular infiltration, cirrhosis, and TNM stage were closely associated with DFS (Table 3). However, multivariate Cox analysis suggested that only miR-21 expression and tumor number were independent predictors of DFS in patients with HCC (Table 3).
TABLE 1: Correlation between miR-21 expression and clinicopathological features.

\begin{tabular}{|c|c|c|c|c|}
\hline \multirow{2}{*}{$\begin{array}{l}\text { Patient } \\
\text { characteristics }\end{array}$} & \multicolumn{4}{|c|}{ miR-21 expression } \\
\hline & Number & $\begin{array}{c}\text { Low } \\
(N=83)\end{array}$ & $\begin{array}{c}\text { High } \\
(N=83)\end{array}$ & $P$ value \\
\hline \multicolumn{5}{|l|}{ Gender } \\
\hline Male & 87 & 48 & 39 & 0.162 \\
\hline Female & 79 & 35 & 44 & \\
\hline \multicolumn{5}{|l|}{ Age (years) } \\
\hline$<55$ & 46 & 25 & 21 & 0.488 \\
\hline$\geq 55$ & 120 & 58 & 62 & \\
\hline \multicolumn{5}{|l|}{ Tumor size $(\mathrm{cm})$} \\
\hline$\leq 5$ & 132 & 74 & 58 & 0.002 \\
\hline$>5$ & 34 & 9 & 25 & \\
\hline \multicolumn{5}{|l|}{ Tumor number } \\
\hline Single & 133 & 72 & 61 & 0.032 \\
\hline Multiple & 33 & 11 & 22 & \\
\hline \multicolumn{5}{|l|}{ Serum AFP $(\mu \mathrm{g} / \mathrm{l})$} \\
\hline$<400$ & 47 & 21 & 26 & 0.389 \\
\hline$\geq 400$ & 119 & 62 & 57 & \\
\hline \multicolumn{5}{|l|}{ Liver cirrhosis } \\
\hline Absence & 38 & 17 & 21 & 0.46 \\
\hline Presence & 128 & 66 & 62 & \\
\hline \multicolumn{5}{|c|}{ Tumor differentiation } \\
\hline Well+moderate & 101 & 63 & 38 & $<0.001$ \\
\hline Poor & 65 & 20 & 45 & \\
\hline \multicolumn{5}{|l|}{ TNM stage } \\
\hline I-II & 96 & 60 & 36 & $<0.001$ \\
\hline III-IV & 70 & 23 & 47 & \\
\hline \multicolumn{5}{|l|}{ Vascular infiltration } \\
\hline Absence & 121 & 66 & 55 & 0.055 \\
\hline Presence & 45 & 17 & 28 & \\
\hline
\end{tabular}

miR-21: microRNA-21; AFP: $\alpha$-fetoprotein.

5.5. Identification and Eligibility of Relevant Studies in the Meta-Analysis. A flow diagram of the study selection process is summarized in Figure 4. Finally, 9 previously published articles and our current cohort study were included in the meta-analysis [17, 18, 26-32]. These eligible studies were published between 2012 and 2019 and included a total of 969 patients with HCC from China, Japan, South Korea, and Greece. These eligible studies were all retrospective cohort studies. 10 studies investigated the relation of miR21 with overall survival (OS) of HCC patients, and 6 explored its connection with disease-free survival (DFS). The method of miR-21 expression detection was all quantitative realtime polymerase chain reaction (qRT-PCR). Characteristics of the eligible studies are summarized in Table 4.

5.6. Meta-Analysis of miR-21 Expression and OS in HCC Patients. Figure 5 shows the forest plot for the survival data. The combined HR was $2.24(95 \% \mathrm{CI}=1.73-2.91, P<0.001)$, indicating that elevated miR-21 expression was significantly predictive of poor OS in HCC patients undergoing surgical 
TABLE 2: Univariate and multivariate Cox regression analysis for OS.

\begin{tabular}{|c|c|c|c|c|}
\hline \multirow{2}{*}{ Variables } & \multicolumn{2}{|c|}{ Univariate analysis } & \multicolumn{2}{|c|}{ Multivariate analysis } \\
\hline & HR (95\% CI) & $P$ value & HR (95\% CI) & $P$ value \\
\hline Age (years) & $1.160(0.556-2.418)$ & 0.693 & & \\
\hline Gender & $0.781(0.412-1.481)$ & 0.45 & & \\
\hline Tumor size & $3.784(1.339-10.700)$ & 0.012 & $3.902(1.273-11.956)$ & 0.017 \\
\hline Tumor number & $2.377(1.217-4.640)$ & 0.011 & & \\
\hline Tumor differentiation & $2.766(1.427-5.361)$ & 0.003 & & \\
\hline TNM stage & $3.232(1.627-6.420)$ & 0.001 & & \\
\hline Vascular infiltration & $2.467(1.273-4.784)$ & 0.007 & $2.56(1.162,5.6399)$ & 0.019 \\
\hline Cirrhosis & $1.971(1.005-3.866)$ & 0.048 & & \\
\hline AFP & $1.731(0.855-3.505)$ & 0.127 & & \\
\hline miR-21 expression & $3.368(1.622-6.993)$ & 0.001 & $2.361(1.016-5.490)$ & 0.046 \\
\hline
\end{tabular}

OS: overall survival; miR-21: microRNA-21; AFP: $\alpha$-fetoprotein; HR: hazard ratio; CI: confidence interval.

TABLE 3: Univariate and multivariate Cox regression analysis for DFS/RFS.

\begin{tabular}{lccc}
\hline Variables & Univariate analysis & Multivariate analysis & HR $(95 \%$ CI $)$ \\
\hline Age (years) & HR $(95 \%$ CI $)$ & $P$ value & 0.62 \\
Gender & $1.201(0.582-2.478)$ & 0.398 & \\
Tumor size & $1.773(0.402-1.437)$ & 0.11 & \\
Tumor number & $2.377(1.082-8.667)$ & 0.035 & \\
Tumor differentiation & $2.994(1.540-5.823)$ & 0.001 & $(1.035-4.862)$ \\
TNM stage & $3.381(1.697-6.733)$ & 0.001 & \\
Vascular infiltration & $2.053(1.078-3.910)$ & 0.029 & \\
Cirrhosis & $2.009(0.374-10.791)$ & 0.416 & \\
AFP & $1.845(1.022-3.329)$ & 0.042 & $2.02(1.042-3.92)$ \\
miR-21 expression & $3.179(1.569-6.442)$ & 0.001 & 0.037 \\
\hline
\end{tabular}

DFS/RFS: disease-free survival/recurrence-free survival; miR-21: microRNA-21; AFP: $\alpha$-fetoprotein; HR: hazard ratio; CI: confidence interval.

resection. The fixed model effect was used to synthesize the data, since no significant heterogeneity existed among these studies $\left(I^{2}=0 \%, P=1.0\right)$.

5.7. Meta-Analysis of miR-21 Expression and DFS/RFS in HCC Patients. Among the eligible studies, 6 referred to the correlation between miR-21 expression and DFS/RFS, including 5 previously published studies and our cohort study. Since recurrence-free survival (RFS) and disease-free survival (DFS) are indicators of disease progression, such as locoregional recurrence or distant metastasis, we merged them for a pooled analysis. Considering that there was no significant heterogeneity between studies $\left(I^{2}=0 \%, P=0.62\right)$, we chose the fixed-effects model to perform the meta-analysis. Forest plots of the meta-analyses for miR-21 expression are depicted in Figure 5. Combined data from eight studies showed that HCC patients with high miR-21 expression had shorter DFS/RFS, with a pooled HR estimate of 2.44 $(95 \% \mathrm{CI}=1.62-3.67, P<0.001)$.

5.8. Publication Bias and Sensitivity Analysis. The funnel plot test was used to evaluate publication bias. As shown in Figures 5 and 6 , the funnel plots were almost symmetric in
OS studies, as well as in DFS/RFS studies. Sensitivity analysis was performed by eliminating the highest weighted study, and there was no individual study that substantially changes the overall HR, which indicates the reliability of our results.

\section{Discussion}

Since the initial recognition of the relation between miR-21 and cancer in 2005 [33], miR-21 has gained wide concern in cancer research for its crucial role in regulating the expression of oncogenes and tumor suppressors. Recently, a series of quantitative analyses have been performed to identify the prognostic role of miR-21 in various cancers. Zhu et al. demonstrated that elevated miR-21 moderately predicts poor overall survival (OS) in general carcinomas $(\mathrm{HR}=1.903$, $95 \% \mathrm{CI}=1.713-2.113, P<0.001)$, especially pancreatic cancer patients [34]. Similar results were summarized in $\mathrm{Hu}$ et al.'s analyses, with pooled HR for OS $2.05(95 \% \mathrm{CI}=1.71-2.46$, $P<0.001$ ) [35]. In gastric cancer, Wang et al. reported higher miR-21 expression could significantly predict poorer survival with pooled $\mathrm{HR}$ for OS $2.00(95 \% \mathrm{CI}=1.39-2.88, P<0.01)$ [36]. However, there were also insignificant or opposite results in some studies. In a meta-analysis of 1,163 non-small-cell 


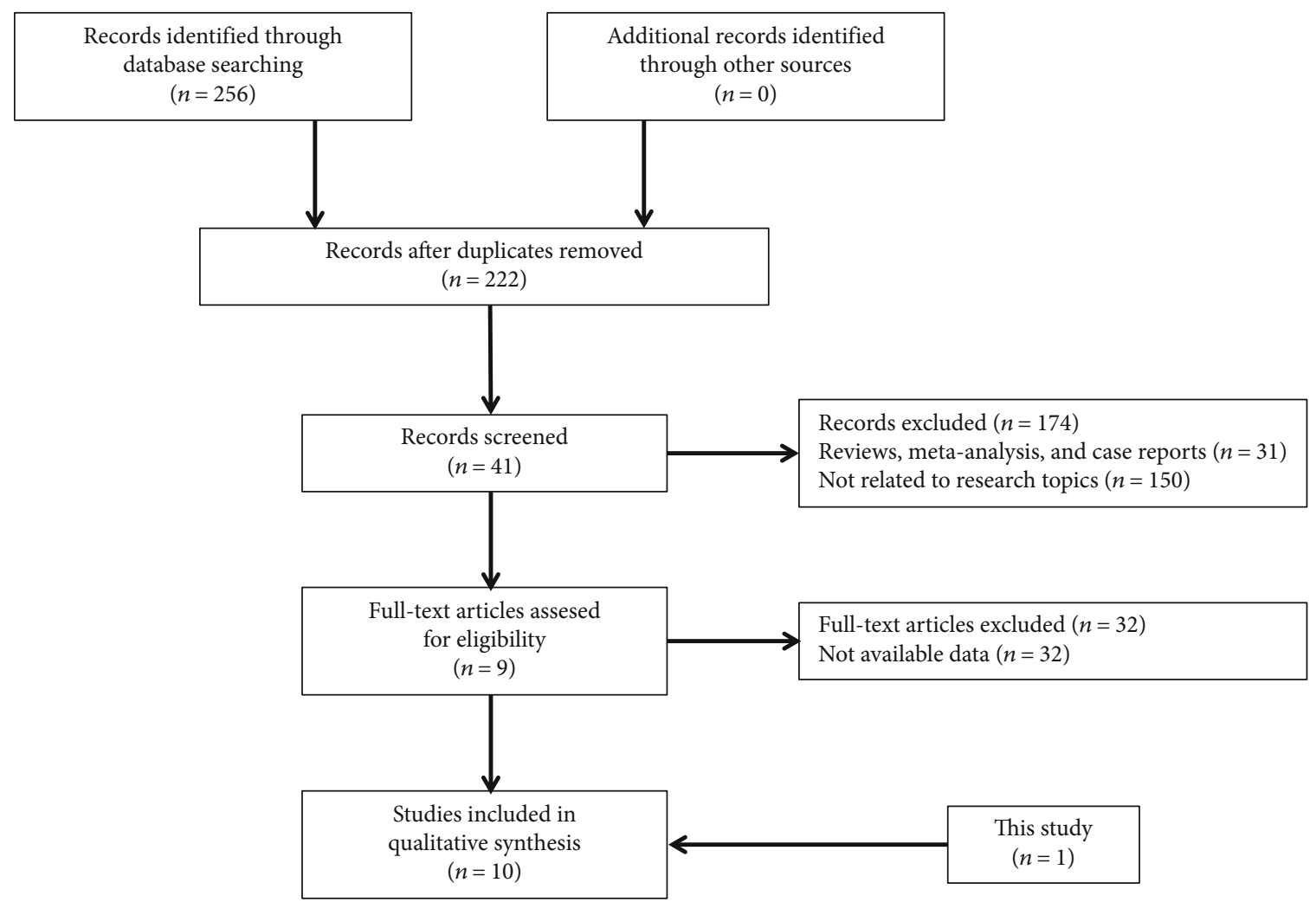

Figure 4: Flow chart of the study selection process.

TABle 4: The characteristics of the pooled studies.

\begin{tabular}{|c|c|c|c|c|c|c|c|c|}
\hline Study (Ref.) & Country & Ethnicity & Sample size & Method & Cutoff & Results & Score (NOS) & Ref. \\
\hline Tomimaru Y 2012 & Japan & Asian & 126 & qRT-PCR & Mean: 0.754 & OS/RFS & 8 & {$[28]$} \\
\hline Karakatsanis A 2013 & Greece & Caucasian & 60 & qRT-PCR & 3.07-fold of control & OS & 7 & [27] \\
\hline Gyongyosi B 2014 & Italy & Caucasian & 20 & qRT-PCR & Median & OS & 6 & {$[25]$} \\
\hline Wang WY 2014 & China & Asian & 119 & qRT-PCR & Median & OS/DFS & 8 & [29] \\
\hline Wang X 2015 & China & Asian & 97 & qRT-PCR & Median & OS & 7 & {$[30]$} \\
\hline Hu L 2016 & China & Asian & 32 & qRT-PCR & Median & OS & 6 & [26] \\
\hline Zhu C 2018 & China & Asian & 50 & qRT-PCR & Median & OS/DFS & 8 & [16] \\
\hline Zhou Y 2018 & China & Asian & 83 & qRT-PCR & Score $\geq 2$ & OS/DFS & 6 & [31] \\
\hline Tian X.P 2019 & China & Asian & 124 & qRT-PCR & Median & OS/RFS & 8 & [18] \\
\hline Our study & China & Asian & 166 & qRT-PCR & Median & OS/DFS & 7 & \\
\hline
\end{tabular}

lung cancer (NSCLC) cases, Ma et al. indicated the HR for OS is $2.19(95 \% \mathrm{CI}:=0.76-6.30, P=0.15)$, which means the miR-21 expression has limited prognostic significance on NSCLC [37]. In general, the prognostic role of miR-21 in cancers is still disputable.

Similarly, different results have been found in studies related to the expression of miR-21 in HCC and its prognostic value. Tian and his colleagues found that the high expression of miR-21 was negatively correlated with the prognosis of patients with HCC after surgery [28]. In another study, Gyongyosi et al. reported that in $20 \mathrm{HCC}$ patients who received sorafenib treatment, the high expression of miR-21 was not an independent predictor of OS [26]. Why is the prognostic significance of miR-21 in HCC patients controversial? First, the treatment of HCC is characterized by the coexistence of multidisciplines and multitreatments, including hepatectomy, liver transplantation, and transarterial chemoembolization (TACE), and different treatments may lead to these differences $[6,38,39]$. Secondly, factors such as region-specific, race-specific, gender-specific, or age-specific factors may affect prognostic outcomes and produce statistical heterogeneity.

Surgical resection is a recommended treatment option in HCC patients with resectable tumor $[4,38]$. Hence, we carried out this two-step study, which focused on thoroughly exploring the relationship between the expression of miR-21 


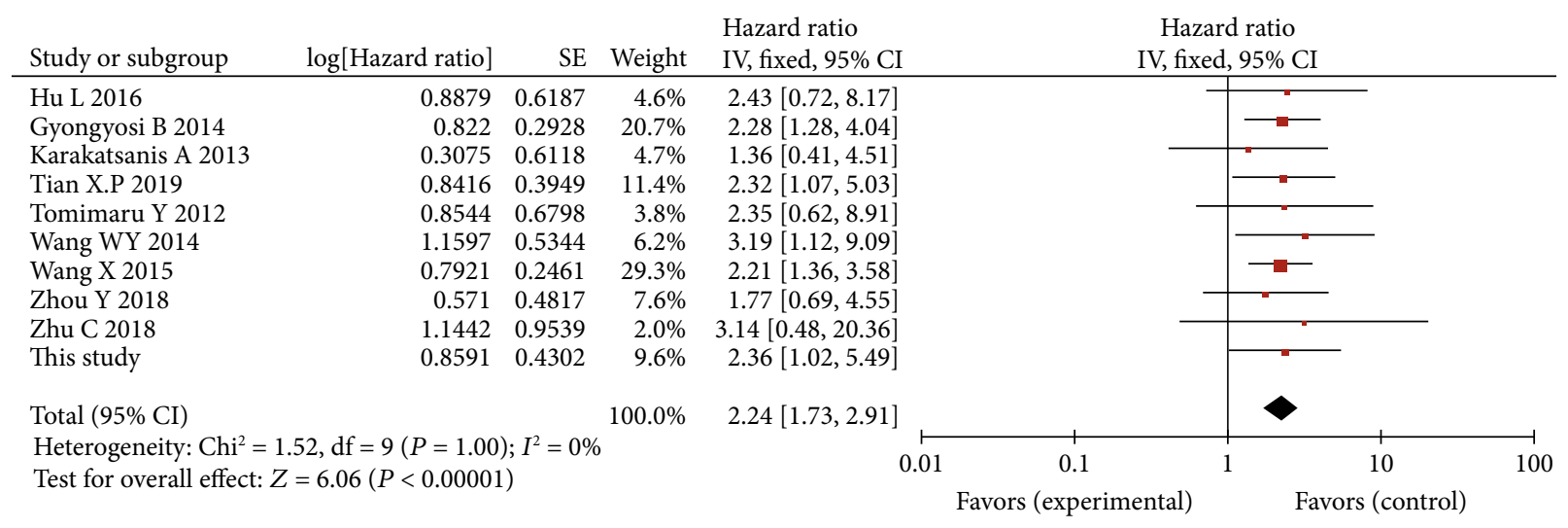

(a)

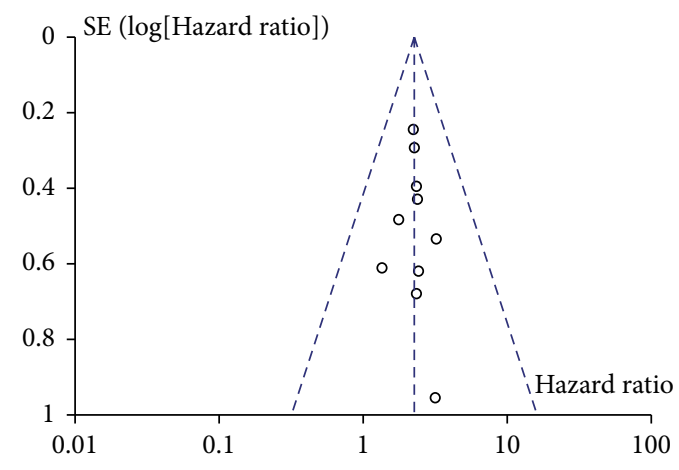

(b)

FIgURE 5: Forest plot (a) and funnel plot (b) for the association between miR-21 expressions with overall survival (OS).

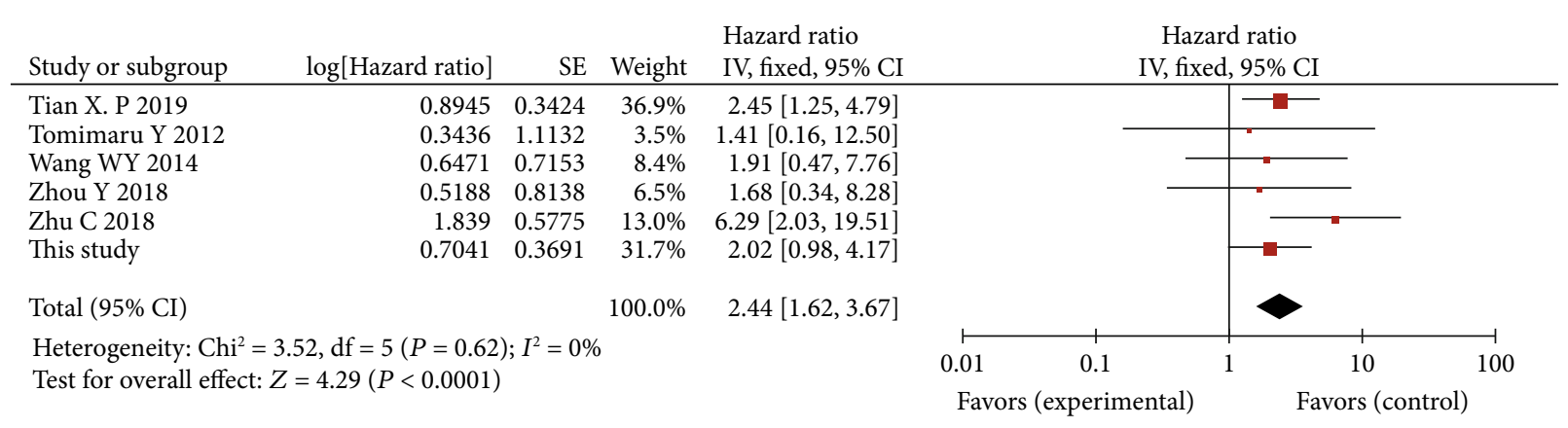

(a)

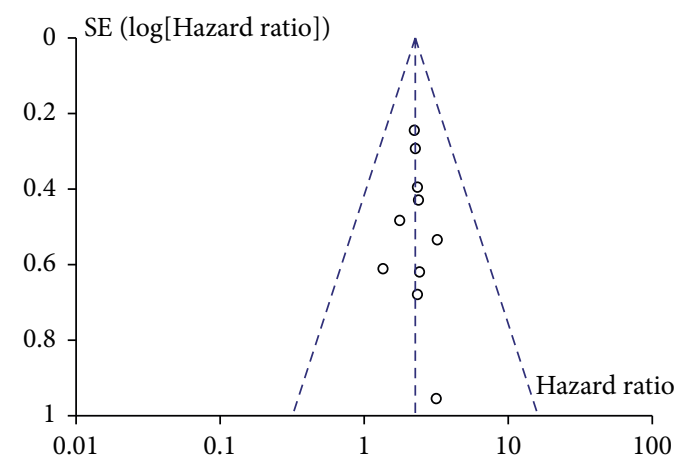

(b)

FIgURE 6: Forest plot (a) and funnel plot (b) for the association between miR-21 expression with recurrence-free survival (RFS)/disease-free survival (DFS). 
and the prognosis of HCC patients who underwent surgical resection. Our current cohort study showed that miR-21 was overexpressed in HCC tissues compared with adjacent normal tissues, and high expression of miR-21 was significantly correlated with shorter OS and DFS/RFS, which was consistent with the subsequent meta-analysis results. In addition, we also evaluated the relationship between the expression of miR-21 and the clinicopathological features of patients with HCC. Our results showed the high expression level of miR-21 was closely related to tumor size, tumor number, tumor differentiation, and TNM stage, suggesting that the expression of miR-21 may be related to the malignant characteristics of the tumor. Our results further clarify the close relationship between the overexpression of miR-21 and the progression, metastasis, and death of HCC.

To our knowledge, this two-part study is the first meta-analysis to thoroughly assess the relationship between miR-21 expression and the prognosis of HCC patients that underwent surgical resection. However, it did have several limitations. First, all eligible studies in the meta-analysis were of a retrospective nature, which might have introduced a degree of bias. Second, the cutoff values were different in various studies. Indeed, there is no common threshold value to define miR-21-positive expression in patients with HCC. Third, when the individual HR together with its variance were not reported in the selected articles, the data are extracted from the survival curve using the method described by Tierney et al. [21]. The estimated HR may be less reliable than the one obtained directly from published statistics.

\section{Conclusion}

Our retrospective cohort study and meta-analysis demonstrated that miR-21 high expression among surgically resected HCC patients is a prognostic factor that indicated adverse survival.

\section{Conflicts of Interest}

The authors declare that they have no conflicts of interest.

\section{Authors' Contributions}

Huang Xujian performed the study design, analysis of data, and manuscript drafting; Xiong Yongfu performed the study design and critical appraisal of the manuscript; Yang Jialin performed the analysis of data; Yang Gang performed data extraction; Li Jingdong performed the critical appraisal of the manuscript and final approval of the manuscript. Each author has approved this version of the manuscript to be published.

\section{Acknowledgments}

This research was funded by the Department of Education of Sichuan Province (Grant No. 16TDD00025).

\section{References}

[1] R. L. Siegel, K. D. Miller, and A. Jemal, "Cancer statistics, 2018," CA: a cancer journal for clinicians, vol. 68, no. 1, pp. 7-30, 2018.

[2] F. Bray, J. Ferlay, I. Soerjomataram, R. L. Siegel, L. A. Torre, and A. Jemal, "Global cancer statistics 2018: GLOBOCAN estimates of incidence and mortality worldwide for 36 cancers in 185 countries," CA: a cancer journal for clinicians, vol. 68, no. 6, pp. 394-424, 2018.

[3] H. B. El-Serag, "Hepatocellular carcinoma," The New England journal of medicine, vol. 365, no. 12, pp. 1118-1127, 2011.

[4] J. K. Heimbach, L. M. Kulik, R. S. Finn et al., "AASLD guidelines for the treatment of hepatocellular carcinoma," Hepatology, vol. 67, no. 1, pp. 358-380, 2018.

[5] A. J. Craig, J. von Felden, T. Garcia-Lezana, S. Sarcognato, and A. Villanueva, "Tumour evolution in hepatocellular carcinoma," Gastroenterology \& hepatology, vol. 17, no. 3, pp. 139$152,2020$.

[6] J. Bruix, L. G. da Fonseca, and M. Reig, "Insights into the success and failure of systemic therapy for hepatocellular carcinoma," Gastroenterology \& hepatology, vol. 16, no. 10, pp. 617-630, 2019.

[7] Y. S. Cheng, X. L. Chen, L. S. Ye et al., "The prognostic significance of NEK2 in hepatocellular carcinoma: evidence from a meta-analysis and retrospective cohort study," Cellular physiology and biochemistry, vol. 51, no. 6, pp. 27462759, 2019.

[8] W. Filipowicz, S. N. Bhattacharyya, and N. Sonenberg, "Mechanisms of post-transcriptional regulation by microRNAs: are the answers in sight?," Nature reviews Genetics, vol. 9, no. 2, pp. 102-114, 2008.

[9] D. P. Bartel, "MicroRNAs: target recognition and regulatory functions," Cell, vol. 136, no. 2, pp. 215-233, 2009.

[10] P. Yang, Q. J. Li, Y. Feng et al., "TGF- $\beta$-miR-34a-CCL22 signaling-induced Treg cell recruitment promotes venous metastases of HBV-positive hepatocellular carcinoma," Cancer Cell, vol. 22, no. 3, pp. 291-303, 2012.

[11] J. Zhou, L. Yu, X. Gao et al., "Plasma microRNA panel to diagnose hepatitis B virus-related hepatocellular carcinoma," Journal of clinical oncology, vol. 29, no. 36, pp. 4781-4788, 2011.

[12] W. Li, X. Dong, C. He et al., "LncRNA SNHG1 contributes to sorafenib resistance by activating the Akt pathway and is positively regulated by miR-21 in hepatocellular carcinoma cells," Journal of experimental \& clinical cancer research : CR., vol. 38, no. 1, p. 183, 2019.

[13] H. Wu, R. Ng, X. Chen, C. J. Steer, and G. Song, "MicroRNA21 is a potential link between non-alcoholic fatty liver disease and hepatocellular carcinoma via modulation of the HBP1p53-Srebp1c pathway," Gut, vol. 65, no. 11, pp. 1850-1860, 2016.

[14] S. Su, Y. Wu, D. Zhu et al., "On-electrode synthesis of shapecontrolled hierarchical flower-like gold nanostructures for efficient interfacial DNA assembly and sensitive electrochemical sensing of microRNA," Small, vol. 12, no. 28, pp. 3794-3801, 2016.

[15] J. du, S. Yang, F. H. Di An et al., "BMP-6 inhibits microRNA21 expression in breast cancer through repressing deltaEF1 and AP-1," Cell research, vol. 19, no. 4, pp. 487-496, 2009.

[16] J. J. Farrell, P. Toste, N. Wu et al., "Endoscopically acquired pancreatic cyst fluid microRNA 21 and 221 are associated with 
invasive cancer," The American journal of gastroenterology, vol. 108, no. 8, pp. 1352-1359, 2013.

[17] C. Zhu, M. Zhang, J. Hu et al., "Prognostic effect of IL6/JAK2/STAT3 signal-induced microRNA-21-5p expression on short term recurrence of hepatocellular carcinoma after hepatectomy," International journal of clinical and experimental pathology, vol. 11, no. 8, pp. 4169-4178, 2018.

[18] A. Karakatsanis, I. Papaconstantinou, M. Gazouli, A. Lyberopoulou, G. Polymeneas, and D. Voros, "Expression of microRNAs, miR-21, miR-31, miR-122, miR-145, miR146a, miR-200c, miR-221, miR-222, and miR-223 in patients with hepatocellular carcinoma or intrahepatic cholangiocarcinoma and its prognostic significance," Molecular carcinogenesis, vol. 52, no. 4, pp. 297-303, 2013.

[19] D. Moher, A. Liberati, J. Tetzlaff, D. G. Altman, and The PRISMA Group, "Preferred reporting items for systematic reviews and meta-analyses: the PRISMA statement," PLoS medicine, vol. 6, no. 7, article e1000097, 2009.

[20] P. R. Williamson, C. T. Smith, J. L. Hutton, and A. G. Marson, "Aggregate data meta-analysis with time-to-event outcomes," Statistics in Medicine, vol. 21, no. 22, pp. 3337-3351, 2002.

[21] J. F. Tierney, L. A. Stewart, D. Ghersi, S. Burdett, and M. R. Sydes, "Practical methods for incorporating summary timeto-event data into meta-analysis," Trials, vol. 8, no. 1, article 16, 2007.

[22] M. K. Parmar, V. Torri, and L. Stewart, "Extracting summary statistics to perform meta-analyses of the published literature for survival endpoints," Statistics in Medicine, vol. 17, no. 24, pp. 2815-2834, 1998.

[23] A. Stang, "Critical evaluation of the Newcastle-Ottawa scale for the assessment of the quality of nonrandomized studies in meta-analyses," European Journal of Epidemiology, vol. 25, no. 9, pp. 603-605, 2010.

[24] D. F. Stroup, J. A. Berlin, S. C. Morton et al., "Meta-analysis of observational studies in epidemiology: a proposal for reporting. Meta-analysis Of Observational Studies in Epidemiology (MOOSE) group," Jama, vol. 283, no. 15, pp. 2008-2012, 2000.

[25] J. P. Higgins, S. G. Thompson, J. J. Deeks, and D. G. Altman, "Measuring inconsistency in meta-analyses," BMJ, vol. 327, no. 7414, pp. 557-560, 2003.

[26] B. Gyöngyösi, É. Végh, B. Járay et al., "Pretreatment microRNA level and outcome in sorafenib-treated hepatocellular carcinoma," Journal of Histochemistry \& Cytochemistry, vol. 62, no. 8, pp. 547-555, 2014.

[27] L. Hu, H. Ye, G. Huang et al., "Long noncoding RNA GAS5 suppresses the migration and invasion of hepatocellular carcinoma cells via miR-21," Tumor Biology, vol. 37, no. 2, pp. 2691-2702, 2016.

[28] X.-P. Tian, C.-Y. Wang, X.-H. Jin et al., "Acidic microenvironment up-regulates exosomal miR-21 and miR-10b in early-stage hepatocellular carcinoma to promote cancer cell proliferation and metastasis," Theranostics, vol. 9, no. 7, pp. 1965-1979, 2019.

[29] Y. Tomimaru, H. Eguchi, H. Nagano et al., "Circulating microRNA-21 as a novel biomarker for hepatocellular carcinoma," Journal of Hepatology, vol. 56, no. 1, pp. 167-175, 2012.

[30] W.-Y. Wang, H.-F. Zhang, L. Wang et al., "miR-21 expression predicts prognosis in hepatocellular carcinoma," Clinics and Research in Hepatology and Gastroenterology, vol. 38, no. 6, pp. 715-719, 2014.
[31] X. Wang, J. Zhang, L. Zhou et al., "Significance of serum microRNA-21 in diagnosis of hepatocellular carcinoma (HCC): clinical analyses of patients and an HCC rat model," International Journal of Clinical and Experimental Pathology, vol. 8, no. 2, pp. 1466-1478, 2015.

[32] Y. Zhou, H. Ren, B. Dai et al., "Hepatocellular carcinomaderived exosomal miRNA-21 contributes to tumor progression by converting hepatocyte stellate cells to cancer-associated fibroblasts," Journal of Experimental \& Clinical Cancer Research, vol. 37, no. 1, p. 324, 2018.

[33] J. A. Chan, A. M. Krichevsky, and K. S. Kosik, "MicroRNA-21 is an antiapoptotic factor in human glioblastoma cells," Cancer Research, vol. 65, no. 14, pp. 6029-6033, 2005.

[34] W. Zhu and B. Xu, "MicroRNA-21 identified as predictor of cancer outcome: a meta-analysis," PLoS One, vol. 9, no. 8, article e103373, 2014.

[35] G.-y. Hu, F. Tao, W. Wang, and K.-w. Ji, "Prognostic value of microRNA-21 in pancreatic ductal adenocarcinoma: a metaanalysis," World Journal of Surgical Oncology, vol. 14, no. 1, article 82, 2016.

[36] Z. Wang, Q. Cai, Z. Jiang, B. Liu, Z. Zhu, and C. Li, "Prognostic role of microRNA-21 in gastric cancer: a meta-analysis," Medical Science Monitor, vol. 20, pp. 1668-1674, 2014.

[37] X. L. Ma, L. Liu, X. X. Liu et al., "Prognostic role of microRNA21 in non-small cell lung cancer: a meta-analysis," Asian Pacific Journal of Cancer Prevention, vol. 13, no. 5, pp. 23292334, 2012.

[38] J. D. Yang, P. Hainaut, G. J. Gores, A. Amadou, A. Plymoth, and L. R. Roberts, "A global view of hepatocellular carcinoma: trends, risk, prevention and management," Nature reviews Gastroenterology \& hepatology, vol. 16, no. 10, pp. 589-604, 2019.

[39] H. J, "Combined TACE and sorafenib for HCC treatment," Nature reviews Gastroenterology \& hepatology, vol. 17, no. 2, p. 66, 2020. 